\title{
Reflets
}

Revue ontaroise d'intervention sociale et communautaire

\section{Principes féministes en milieu coopératif}

\section{Lyne Bouchard}

Volume 3, numéro 2, automne 1997

Visibles et Partenaires : Pratiques et recherches féministes

URI : https://id.erudit.org/iderudit/026178ar

DOI : https://doi.org/10.7202/026178ar

Aller au sommaire du numéro

Éditeur(s)

Reflets : Revue ontaroise d'intervention sociale et communautaire

ISSN

1203-4576 (imprimé)

1712-8498 (numérique)

Découvrir la revue

Citer cet article

Bouchard, L. (1997). Principes féministes en milieu coopératif. Reflets, 3(2), 156-165. https://doi.org/10.7202/026178ar

Tous droits réservés (C) Reflets : Revue ontaroise d'intervention sociale et communautaire, 1997

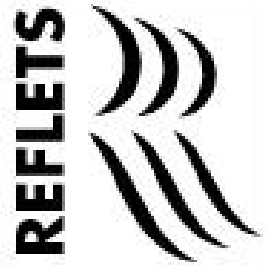

Ce document est protégé par la loi sur le droit d'auteur. L'utilisation des services d'Érudit (y compris la reproduction) est assujettie à sa politique d'utilisation que vous pouvez consulter en ligne.

https://apropos.erudit.org/fr/usagers/politique-dutilisation/ 


\section{Principes féministes en milieu coopératif}

D'emblée, je tiens à préciser que le coopératisme est un outil intéressant à explorer comme féministe. Le coopératisme n'est pas un but en soi, mais peut s'avérer un mécanisme potentiel de gestion féministe.

\section{Lyne Bouchard ${ }^{1}$}

Coopératrice à La coopérative Convergence inc., Ottawa

\section{Introduction}

Dans cet article, je me pencherai sur une pratique de gestion qui s'est élaborée progressivement au fil d'une expérience en milieu coopératif de plus de dix ans. Cette pratique, et surtout sa construction théorique, fait suite à un travail de réflexion entrepris par les coopératrices au cours de la dernière année. En effet, dans le cadre d'une démarche collective, les coopératrices ont voulu faire la théorie de leurs pratiques passées et des valeurs qui avaient guidé jusqu'alors leur action, en énoncant les principes féministes de gestion d'une coopérative.

En premier lieu, je présenterai les principes coopératifs et décrirai brièvement ce qu'est la coopérative Convergence. Dans un second temps, je ferai état du processus de réflexion théorique portant sur les pratiques de gestion d'une coopérative de travail de femmes francophones. À cette fin, j'examinerai les transformations que la coopérative a connues au fil des ans, et plus précisément, les étapes de la dernière transformation, vécue au 
cours de l'année 1996-1997. Enfin, je consacrerai une large place à la discussion des principes qui encadrent nos pratiques et notre gestion, dont deux aspects me semblent particulièrement révélateurs, soit l'autogestion et les principes féministes.

\section{Principes coopératifs}

Il y a sept principes qui gouvernent le travail de toutes les coopératives à l'échelle mondiale. Ces principes ont été formulés à Manchester en 1995 par l'Alliance coopérative internationale. Ces principes sont :

1) l'adhésion libre et volontaire;

2) le pouvoir démocratique des membres;

3) la participation économique des membres;

4) l'autonomie et l'indépendance de la coopérative;

5) l'éducation, la formation et l'information;

6) la coopération avec les autres coopératives; et

7) l'engagement envers la communauté.

\section{Coopérative Convergence inc.}

La coopérative Convergence est une entreprise de consultation travaillant dans les domaines de la recherche, de la formation, de la rédaction, et de la planification stratégique auprès d'organismes de femmes, communautaires ou gouvernementaux. Si elle opère comme entreprise enregistrée depuis 1984 et fonctionne sous le mode de coopérative depuis 1987, elle ne s'est officiellement incorporée en coopérative de travail qu'en $1991^{2}$.Ainsi, la réflexion exprimée ici est le fruit de plus de dix ans d'expérience coopérative guidée par l'action.

À l'origine, l'entreprise n'était formée que de trois associées. Ainsi, la première transformation qu'a connue l'entreprise, en 
1987, a été d'ouvrir la membriété à d'autres femmes et de se constituer légalement en coopérative, puis en coopérative de travail francophone. Dans un premier temps, nous avons cherché à définir le fonctionnement interne de la coopérative et à établir les mécanismes de gestion et de viabilité. Dans un deuxième temps, les coopératrices ont multiplié les efforts afin de s'ajuster aux soubresauts du marché de la consultation et d'offrir des produits toujours accessibles et de qualité ${ }^{3}$.

La deuxième transformation a eu lieu en 1996, soit après dix ans de fonctionnement interne et de pratique efficace. Dotée d'une solide réputation, les coopératrices ressentaient pourtant le besoin de confirmer la raison d'être de la coopérative et de valider les pratiques qui l'encadraient. C'est à ce moment qu'une démarche de transformation appelée peau neuve est amorcée.

\section{Démarche de théorisation}

L'opération peau neuve nous a permis d'entreprendre une démarche de réflexion théorique de nos pratiques de gestion féministe. En effet, durant l'été et l'automne 1996, les coopératrices se sont rencontrées à plusieurs reprises afin de préciser et de valider la raison d'être de la coopérative.

De plus, nous voulions offrir le fruit de notre réflexion à un plus grand nombre de femmes. Nous avons donc organisé une rencontre, le samedi 15 février 1997, où plus de seize femmes provenant de divers milieux, en majorité des femmes liées au réseau de la coopérative, se sont réunies. Cette rencontre, où nous avons présenté la vision et l'identité de Convergence, a été l'occasion d'explorer le potentiel de Convergence, tout en vérifiant l'intérêt des participantes en vue d'un recrutement éventuel.

Ainsi, les femmes présentes ont conclu que Convergence pouvait répondre aux besoins des femmes en partageant ses ressources, ses idées et son information. Quant aux orientations futures, les participantes ont mentionné qu'il faudrait chercher à développer 
et à structurer des réseaux, et que Convergence pourrait oeuvrer à la coordination et à la systématisation de ces réseaux. De plus, la coopérative pourrait chercher à démystifier l'informatique, offrir une plus grande variété de services, devenir plus visible, créer une sorte de "guichet unique» en matière de réseautage d'information pour les femmes et augmenter le nombre de ses coopératrices.

En effet, nous avons réitéré l'importance de consolider le coeur de la coopérative, soit son noyau de travailleuses. Nous n'étions plus que trois travailleuses dont une devait quitter au cours des prochains mois. Nous voulions donc recruter un minimum de deux ou trois nouvelles coopératrices. Un processus de recrutement fut entrepris à cette occasion et, depuis avril 1997, deux coopératrices se sont jointes au noyau en place.

\section{Principes encadrant nos pratiques et notre gestion}

"L'autogestion signifie le partage entre les coopératrices de l'ensemble des tâches de gestion de la coopérative...”
J'aborderai dans cette section deux aspects de notre gestion qui sont à la base de notre coopérative, soit les principes d'autogestion et les principes féministes. Si l'autogestion impose une façon de faire différente de toute autre forme de gestion, les principes féministes caractérisent notre coopérative par son approche et son orientation particulière. Ces deux aspects complémentaires suggèrent une réflexion continue qui mène à l'action.

\section{Autogestion}

L'autogestion peut être envisagée selon deux points de vue, soit selon ce qu'elle favorise, soit selon ce qu'elle exige comme responsabilités. L'autogestion signifie le partage entre les coopératrices de l'ensemble des tâches de gestion de la coopérative (comptabilité, élaboration de contrats, fonctionnement interne, etc.). Ces tâches de gestion ne different pas de celles qu'on retrouve dans toutes entreprises. De l'administration à la gestion financière, tout y passe. Ainsi, les cinq coopératrices sont automatiquement 
les cinq gestionnaires de l'entreprise. Si l'ensemble du travail d'administration et de gestion est partagé entre les cinq femmes, sa répartition répond davantage à des principes d'équité que d'égalité. En effet, la distribution des responsabilités et des tâches s'établit selon les expertises, les disponibilités et les intérêts de chacune.

De par le partage des responsabilités et des tâches de gestion, l'autogestion favorise une meilleure circulation de l'information et donc, un meilleur équilibre du pouvoir. Par exemple, une coopératrice peut être désignée comme "responsable du développement interne» de la coopérative, bien qu'elle ne fasse, en réalité, qu'une partie des tâches relevant de ce secteur.Ainsi, ce mode de fonctionnement permet, premièrement, à plusieurs femmes de détenir l'information sur un sujet donné, et deuxièmement, d'éviter qu'une seule d'entre elles n'ait l'entière responsabilité d'un dossier.

L'autogestion oblige également les coopératrices à se doter de mécanismes d'imputabilité entre elles et envers la coopérative. Cette obligation relève du principe collectif de partage des responsabilités. Elle impose une discipline individuelle et collective à l'égard des responsabilités de chacune et de la bonne marche de l'organisme. Nos mécanismes de gestion mis en place dans la coordination, le partage d'information ou la prise de décision sur certains dossiers, ont souvent été le fruit de cette obligation.

\section{Principes féministes}

Durant l'exercice de transformation peau neuve en 1996-1997, les coopératrices ont établi, d'un commun accord, huit principes féministes de coopération. Ces principes, qui encadrent nos pratiques et notre gestion, sont féministes car ils résultent d'une réflexion collective sur une expérience en milieu coopératif féministe. Et j'insiste sur ce point. Ces principes sont féministes parce qu'ils témoignent du vécu de femmes engagées dans le coopératisme et qu'ils sont le reflet de leurs valeurs, de leurs méthodes de travail, de leurs visions de l'expérience coopérative. J'illustrerai, dans ce qui suit, chaque principe, sans toutefois établir un ordre d'importance entre eux. 
«On travaille

activement à l'épuration de la hiérarchie dans la membriété, les mécanismes décisionnels et la distribution des biens.»

«La flexibilité s'exprime par des efforts d'adaptation tant à la clientèle qu'aux méthodes de travail et au rythme de travail des coopératrices.»
Les intérêts collectifs priment sur les intérêts individuels ${ }^{4}$ : La Coopérative vise, comme objectif principal, à créer du travail rémunéré pour les femmes. Ce principe implique que nous cherchions à fidéliser les clients à la coopérative et non à une coopératrice en particulier, bien que celle-ci exécute la tâche. De ce fait, une collectivité de femmes se prend en main et collabore à se créer du travail.

On travaille activement à l'épuration de la hiérarchie dans la membriété, les mécanismes décisionnels et la distribution des biens ${ }^{5}$. Ce principe se traduit notamment par la reconnaissance des mêmes droits et privilèges à une nouvelle coopératrice qu'à une femme qui est là depuis plus dix ans.Ainsi la prise de décisions nécessite le consensus de toutes.

L'autonomie et l'entraide sont développées parallèlement tant au sein de la coopérative que chez ses membres. ${ }^{6}$. Lorsqu'une coopératrice est responsable d'un dossier ou d'une tâche particulière, elle détient une grande liberté d'action pour mener à bien le dossier. Cependant, elle peut à tout moment demander ou bénéficier de l'appui d'une ou de plusieurs de ses collègues. Par ailleurs, lorsqu'une coopératrice doit interrompre son travail et s'absenter, les autres collègues prennent la relève et assument la continuité du travail. À son retour, les dossiers ne se sont pas accumulés.

La flexibilité s'exprime par des efforts d'adaptation tant à la clientèle qu'aux méthodes de travail et au rythme de travail des coopératrices ${ }^{7}$. Ce principe se concrétise, entre autres, au niveau du fonctionnement interne de la coopérative par la liberté de choisir ses horaires et ses charges de travail.En effet, les coopératrices ne sont pas soumises à un quota de chiffre d'affaires. De plus, elles pratiquent leur travail d'experte-conseil selon une approche qui cherche à remettre le pouvoir au groupe client. Cela exige une attitude flexible, une capacité d'adaptation envers nos clients. Loin de nous présenter comme expertes détenant toutes les solutions, nous accompagnons le groupe client dans une démarche visant à lui rendre sa propre capacité d'action.

La culture d'expression est marquée tant par l'usage de la parole que par la construction de la mémoire collective du groupe $e^{8}$ Les retraites annuelles d'hiver et d'été sont des moments privilégiés de prise de parole et d'échange et incarnent de façon particulière ce 
«L'inclusion

s'applique tant à la gestion interne qu'au travail effectué à l'extérieur de la coopérative et vise à mettre fin aux stéréotypes et aux injustices sociales.»

"L'engagement face à la communauté est une valeur intrinsèque...» principe ${ }^{9}$. Ces moments sont alors l'occasion d'apporter des changements de toutes sortes à la coopérative. Pourtant, je ne cacherai pas que la mise en place de cette culture d'expression au quotidien pose de grands défis à l'équipe de coopératrices. En effet, le manque de temps et la lourde tâche de chaque coopératrice constituent des barrières bien réelles à l'établissement d'un processus de partage et d'échanges quotidiens, et ce bien que nous cherchions toujours les moyens d'y apporter une solution. Par ailleurs, notre participation, nos conférences présentées lors de divers colloques provinciaux, nationaux et internationaux, permettent non seulement de documenter notre cheminement et nos questionnements face à nos pratiques et à notre gestion, mais contribuent également à la mémoire collective du mouvement des femmes.

L'inclusion s'applique tant à la gestion interne qu'au travail effectué à l'extérieur de la coopérative et vise à mettre fin aux stéréotypes et aux injustices sociales ${ }^{10}$. Dans nos rapports auprès des groupes, nous cherchons à promouvoir un principe d'inclusion, c'est-à-dire à amener les personnes et les collectivités à collaborer ensemble et à établir des liens qui intègrent et dépassent leurs différences, voire leurs multiples appartenances: milieux, identités, classes sociales, etc.Ainsi, la coopérative a pris l'initiative de développer des outils de sensibilisation, entre autres, à l'oppression des francophones et à celle des lesbiennes. Et nous n'hésitons pas à communiquer clairement notre vision à nos clients sur ces deux inégalités sociales. Par ailleurs, si les membres de la coopérative travaillent activement à confronter ces inégalités, il nous reste encore à développer un réseau d'associées plus représentatif de la diversité des communautés francophones. Sur ce dernier point, des efforts ont été entrepris, bien qu'ils n'aient pas donné les résultats escomptés jusqu'à présent.

L'engagement face à la communauté est une valeur intrinsèque de la coopérative comme de ses membres de façon individuelle ${ }^{11}$. Chaque coopératrice est militante dans un milieu donné. Nos membres sont activement et bénévolement engagées, auprès des femmes, dans les secteurs de la santé, de la violence, de l'employabilité, de l'orientation sexuelle et du mouvement féministe francophone. 
Notre processus de sélection incarne ce principe d'engagement car, pour devenir coopératrice, les candidates ont à présenter leurs lieux de militantisme et leurs intérêts quant au changement social. Aussi, la coopérative est membre active à la Table féministe francophone de concertation provinciale de l'Ontario.

Le dernier principe global veut que l'on travaille activement et continuellement à établir un juste équilibre dans l'application de ces principes $^{12}$. À cette fin, nos rencontres bi-hebdomadaires sont l'occasion de discuter et de modifier nos approches au fur et à mesure que les membres perçoivent un problème ou qu'elles questionnent une façon de faire ou une décision.

\section{Conclusion}

Bien que nous ayons une grande autonomie dans notre travail, un partage collectif de responsabilités, la liberté de contrôler notre temps, notre horaire et nos méthodes de travail, n'allez surtout pas croire que la vie d'une coopératrice est un paradis pour autant. Les engagements financiers, moraux et sociaux sont parfois lourds à porter.

Nous avons vécu des étapes de consolidation interne au niveau de notre fonctionnement et de notre structure. Nous avons traversé les soubresauts du marché de la consultation. Nous avons validé et confirmé nos pratiques et notre vision. Après avoir vécu une modification de sa membriété par l'ajout de deux nouvelles coopératrices, nous considérons que les prochaines années seront très importantes. En effet, elles permettront à la nouvelle équipe en place, et peut-être à d'autres coopératrices, de peaufiner non seulement nos pratiques, mais surtout de s'ouvrir à d'autres milieux. Ainsi, la réflexion sur nos pratiques de gestion féministe et de gestion tout court se poursuivra sans cesse, et saura ultimement enrichir les coopératrices ainsi que les femmes qu'elles desservent. 


\section{Bibliographie}

BOUCHARD, Lyne et al. (1992). «Une coopérative de travail: une possibilité à explorer», dans Thérèse Boutin et Christiane Rabier (dir. de publ.), Actes du colloque Les femmes francophones en milieu minoritaire. État de la recherche, Sudbury, Institut franco-ontarien, 113-121.

BOUCHARD, Lyne (1993). «Convergence : l'expérience féministe du coopératisme», dans Linda Cardinal (dir. de publ.), Une langue qui pense. La recherche en milieu minoritaire francophone au Canada, Ottawa, Éditions de l'Université d'Ottawa, 34-42.

BOUCHARD, Lyne (1996). «La coopérative Convergence», dans À partir de notre expérience: Femmes de la francophonie ontarienne, Cahiers réseau de recherches féministes, Montréal, Presses de l’Université du Québec à Montréal.

BOUCHARD, Lyne (à paraître). «La gestion féministe et le coopératisme : y a-t-il des liens?», dans Colloque international: La recherche féministe dans la francophonie. État de la situation et piste de collaboration, Québec, Université Laval.

LA COOPÉRATIVE CONVER GENCE (1997). Projet de modifications de l'Acte d'association, Ottawa, Coopérative Convergence.

LA COOPÉRATIVE CONVER GENCE (1996-1997). Comptes-rendus des rencontres des 19 juin, 3 et 26 juillet, 12 et 26 août, 2 et 23 septembre, 5 et 20 novembre, 16 décembre 1996 et des 7 et 13 janvier, 10 et 15 février 1997, dans le cadre de l'opération «peau neuve», Ottawa, Coopérative Convergence.

\section{Notes}

1. Je remercie mes collègues Chantal Cholette, Geneviève Guidon et Blanche Paradis pour leur collaboration à l'écriture de ce texte. Je tiens à remercier tout spécialement Chantal Chollette qui l'a présenté à ma place lors du colloque Visibles et Partenaires.

2. Pour de plus amples détails sur la création de la coopérative, voir la bibliographie ci-dessus.

3. La Coopérative Convergence a son siège social dans la région d'Ottawa. Or, on se souviendra qu'à la fin des années 1980, on a assisté à des licenciements massifs dans la fonction publique fédérale. Non seulement ce genre de phénomène amène-t-il sur le marché de la consultation, une vaste population de pigistes, mais il exacerbe la concurrence et rend plus difficile l'obtention de contrats.

4. Extrait de la modification de l'Acte d'association de La coopérative Convergence inc., Ottawa, juin, 1997.

5. Idem.

6. Idem.

7. Idem.

8. Idem. 
9. Les retraites sont des rencontres internes de ressourcement entre les coopératrices où nous faisons un bilan et une planification de nos pratiques et de notre gestion quotidienne. Pour de plus amples détails, veuillez consulter la bibliographie ci-dessus. 10. Idem.

11. Idem.

12. Idem. 